\title{
Disaster situations and psychiatric disorders
}

\author{
D Aryasinghe, SS Williams
}

Posttraumatic stress symptoms, co-morbid psychiatric symptoms and distorted cognitions among flood victims of different ages (1)

This is a study examined PTSD symptoms among flood victims. The aims of the study were to investigate whether flood-related PTSD, psychiatric co-morbidity and cognitive distortions would differ according to age and distinctive patterns of association between the preceding variables for different age groups.

One hundred and fifty-four flood victims of different ages completed standardised questionnaires measuring PTSD, psychiatric co-morbidity and cognitive distortions. Adolescents and young adults reported significantly fewer PTSD, psychiatric co-morbidity and distorted cognition symptoms than people who were older. Preoccupation with danger and hopelessness were associated with both outcomes for adolescents, people in their thirties and middle-aged/older people. For young adults, helplessness was associated with PTSD; hopelessness and preoccupation with danger with psychiatric co-morbidity.

This study suggests that adolescents and young adults buffered against flood-related psychological distress better than older people. Distorted cognitions related to distress outcomes appear to differ depending on age.

Prevalence and risk factors for posttraumatic stress reaction among resident survivors of the tsunami that followed the great east Japan earthquake, March 11, 2011(2)

The aims of the authors were to study the prevalence and risk factors of post-traumatic stress reaction (PTSR) among the resident survivors of the tsunami triggered by the great east Japan earthquake, on March 11, 2011. They carried out a cross-sectional household screening for health needs among the survivors 2-4 months after the tsunami. Questions assessing PTSR were included in the screening interviews.
Five thousand one hundred and three survivors were interviewed and 5.7\% experienced PTSR. The risk factors for developing PTSR differed according to the height of the house flooding; when flooding occurred below the ground floor, PTSR was significantly associated with being female and having been prescribed regular psychotropic medication prior to the tsunami. In addition, being middle aged and living alone were also risk factors when flood levels reached above the ground floor.

The authors concluded that the prevalence of PTSR among the survivors to be high and suggested that people already prescribed psychotropic medication, their families and people who live by themselves may require more attention and support following a disaster of this nature.

The relationship between psychiatric morbidity and quality of life: interview study of Norwegian tsunami survivors 2 and 6 years post-disaster (3)

The study investigated the impact of psychiatric disorders on Quality of Life (QOL) cross-sectionally and longitudinally, in a group of Norwegian tourists severely exposed to the 2004 tsunami.

Sixty-two adult Norwegian tsunami survivors were interviewed face to face 2 years post-tsunami (T1) and 58 were interviewed again by telephone 6 years posttsunami (T2). The majority (81\%) reported direct exposure to the waves, and 14 participants (23\%) lost a close family member in the tsunami. Psychiatric morbidity was measured by structured clinical interviews and QOL was assessed using the WHO Quality of Life-Bref scale. Multiple linear regression analyses were performed to assess the independent effects of psychiatric disorders on QOL 2 and 6 years after the tsunami.

The most prevalent psychiatric disorders 2 years after the traumatic event were anxiety disorders, depression and PTSD; and there was significant co-morbidity between depression and PTSD. Psychiatric disorders, especially depression, and also PTSD and other anxiety disorders, were associated with reduced QOL. Psychiatric disorders were more strongly related to QOL at 6 years after the tsunami than at 2 years. Limitations of the study 
include the small sample size, and the fact that assessment was conducted via telephone rather than by face-to-face interview.

The overall findings suggest that psychiatric disorders, especially depression, is related to reduced QOL in a disaster exposed population. Post-disaster psychiatric disorders, such as PTSD and especially depression, should be comprehensively addressed in the aftermath of disasters.

Culture, local capacity, and outside aid: a community perspective on disaster response after the 2009 tsunami in American Samoa (4)

Research on diverse cultural contexts has indicated that aid organisations often fail to leverage local, culturallygrounded resources and capacities in disaster-affected communities. A case-study methodology was employed to explore the relationship between local and external disaster response efforts in American Smoa, following the earthquake and tsunami on 29 September 2009 in the southern Pacific Ocean, with a specific focus on the role of culture in defining that relationship. Interview and focus group data from 37 participants, along with observational data, suggested that the local response to the event was swift and grounded in Samoan cultural systems and norms. External aid was viewed as helpful in some respects, although, on the whole, it was seen as a disruption to village hierarchies, social networks, and local response efforts. The study discusses the implications for the role of outside aid in diverse cultural contexts, and makes suggestions for improving the ecological fit of post-disaster interventions.

Exposure to conflict and disaster: A national survey on the prevalence of psychotic experiences in Sri Lanka (5)

This is an interesting study that looked at the possibility of persons exposed to trauma having an increased propensity to psychotic experiences. This study aimed to investigate the prevalence of psychotic experiences in a general population sample in Sri Lanka and associations with conflict- and tsunami-related trauma.

A cross-sectional, multi-stage, cluster sampling design was used to estimate the prevalence of psychotic symptoms. Data on socio-demographic characteristics, conflict- and tsunami-related trauma, and psychotic experiences were collected using culturally adapted measures in a sample of 5927 participants. The weighted prevalence of psychotic symptoms was 9.7\%. Exposure to one or more conflict-related events (adjusted OR (aOR) $1.79,95 \%$ CI $1.40-2.31, \mathrm{p}<0.001$ ) and loss or injury of a family member or friend through conflict (aOR, 1.83, 95\%
CI 1.42-2.37, $\mathrm{p}<0.001$ ) were associated with increased odds of reporting psychotic experiences.

Psychotic experiences were more common in individuals directly exposed to tsunami disaster (aOR, 1.68, 95\% CI 1.04-2.73, $\mathrm{P}=0.035$ ) and in those who had a family member who died or was injured as result of tsunami (aOR, 1.42, 95\% CI 1.04-1.94, $\mathrm{p}=0.029)$. Limitations of the study include a possible selection bias, potential issues due to cross cultural validity of the tools used, and the fact that confounders such as genetic risk and cannabis use could not be excluded.

However this is a large study and the first to explore population prevalence of psychotic experiences in the Sri Lankan population. The overall findings suggest that exposure to traumatic events in armed conflicts and natural disasters may be important socio-environmental factors in the development of psychotic experiences, and the authors suggest further research to delineate the pathways by which such experiences may occur, following exposure to traumatic events.

\section{The neural correlates of memory for a life- threatening event: An fMRI study of passengers from flight AT236 (6)}

The authors compared the neural correlates of remote re-experiencing of traumatic events in survivors of the near crash of Air Transat (AT) Flight 236 and a group of non-traumatized individuals. Brain activity was monitored during video-cued recollection of the disaster, the September 11 attack in 2001 and a non-emotional (neural) event.

The survivors showed a robust memory enhancement effect for the AT incident compared to the latter two events. The traumatic enhancement was associated with activation in the amygdale, medial temporal lobe, anterior and posterior midline, and visual cortex. This brainbehaviour relationship was also seen in relation to 9/11 in the survivors. This pattern was not observed in the comparison group, which suggests that remote traumatic memory is mediated by activity of the amygdala, which likely enhances vividness of the memory, through its influence on hippocampal and ventral visual systems.

\section{Declaration of interest}

None declared

D Ariyasinghe, Department of Psychiatry, Faculty of Medicine, University Peradeniya

SS Williams, Department of Psychiatry, Faculty of Medicine, University Kelaniya

Corresponding author: D Ariyasinghe

Email: dewasmika.ariyasinghe@gmail.com 


\section{References}

1. Chung MC, Jalal S, Khan NU. Posttraumatic stress symptoms, co-morbid psychiatric symptoms and distorted cognitions among flood victims of different ages. J Ment Health 2016; 3: 1-8.

2. Matsubara C, Murakami H, Imai K, et al. Prevalence and Risk Factors for Post-Traumatic Stress Reaction Among Resident Survivors of the Tsunami That Followed the Great East Japan Earthquake, March 11, 2011. Disaster Med Public Health Prep 2016 Apr 14:1-8.

3. Hussain A, Nygaard E, Siqveland J, Heir T. The relationship between psychiatric morbidity and quality of life: interview study of Norwegian tsunami survivors 2 and 6 years postdisaster. BMC Psychiatry 2016; 31; 16: 173.

4. Binder SB, Baker CK. Culture, local capacity, and outside aid: a community perspective on disaster response after the 2009 tsunami in American Samoa. Disasters. 2016.

5. Keraite A, Sumathipala A, Siriwardhana C, Morgan C, Reininghaus U. Exposure to conflict and disaster: A national survey on the prevalence of psychotic experiences in Sri Lanka. Schizophr Res 2016; 171(1-3): 79-85.

6. Palombo DJ, McKinnon MC, McIntosh AR, Anderson AK, Todd RM, Levine B. The neural correlates of memory for a life-threatening event: An fMRI study of passengers from flight AT236. Clin Psychol Sci 2016; 4(2): 312-9. 